\title{
Telegraph Noise and Fractional Statistics in the Quantum Hall Effect
}

\author{
C.L. Kane \\ Dept. of Physics, University of Pennsylvania, Philadelphia, PA 19104
}

\begin{abstract}
We study theoretically nonequilibrium noise in the fractional quantum Hall regime for an Aharonov Bohm ring which has a third contact in the middle of the ring. We show that as a consequence of their fractional statistics the tunneling of a Laughlin quasiparticle between the inner and outer edge of the ring changes the effective Aharonov Bohm flux experienced by quasiparticles going around the ring, leading to a change in the conductance across the ring. A small current in the middle contact therefore gives rise to fluctuations in the current flowing across the ring which resemble random telegraph noise. We analyze this noise using the chiral Luttinger liquid model. At low frequencies the telegraph noise varies inversely with the quasiparticle tunneling current, and can be much larger than the shot noise. We propose that combining the Aharonov Bohm effect with a noise measurement provides a direct method for observing fractional statistics.
\end{abstract}

PACS numbers: PACS numbers: 73.43.Jn, 73.50.Td, 71.10.Pm

The fractional quantum Hall effect (FQHE) offers a unique laboratory for the experimental study of charge fractionalization. At filling $\nu=1 / m$ the FQHE state supports quasiparticles with charge $-e / m[1]$. Shortly after Laughlin's pioneering explanation of the FQHE, Halperin 2] pointed out that in addition to having fractional charge, Laughlin quasiparticles (LQP's) obey fractional statistics. As elaborated further by Arovas, Schrieffer and Wilczek[3], when two LQPs are adiabatically interchanged in the plane, the many particle wavefunction picks up a quantum mechanical phase $\Theta_{m}=\pi / m$. Equivalently, when one LQP is transported around another a statistical phase $2 \Theta_{m}$ is acquired. LQPs are thus neither bosons nor fermions, but rather more general particles, dubbed anyons by Wilczek 4$]$.

Shot noise experiments using a quantum point contact (QPC) setup suggested earlier [5] allowed de-Piccioto et al. [6] and Saminadayar et al. 7] to perform a direct measurement of the fractional charge of the LQP. To date there has been no similarly direct observation of the fractional statistics of the LQP. Such an observation requires a quantum interference measurement to extract the statistical phase. Proposed experiments have focused on the equilibrium Aharonov Bohm (AB) effect 8, 9, 10]. More recently, Safi et al. 11] have suggested a three terminal Hanbury-Brown Twiss type noise experiment. In this letter we propose a method for directly probing the statistical phase by combining the $\mathrm{AB}$ effect with a noise measurement. For our geometry fractional statistics leads to a qualitatively new kind of noise, which resembles random telegraph noise.

This work was inspired by a recent experiment in which Ji et al. 12 constructed an electronic Mach-Zehnder interferometer by fabricating a ring shaped quantum Hall sample with QPCs along with a lead inside the ring. We propose a variant on their geometry shown in Fig. 1. QPCs 1 and 2 allow LQPs on the outer edge to circle the ring, leading to an $\mathrm{AB}$ effect in the current flowing between leads 1 and 2. QPC3 allows LQPs to tunnel be- (a)

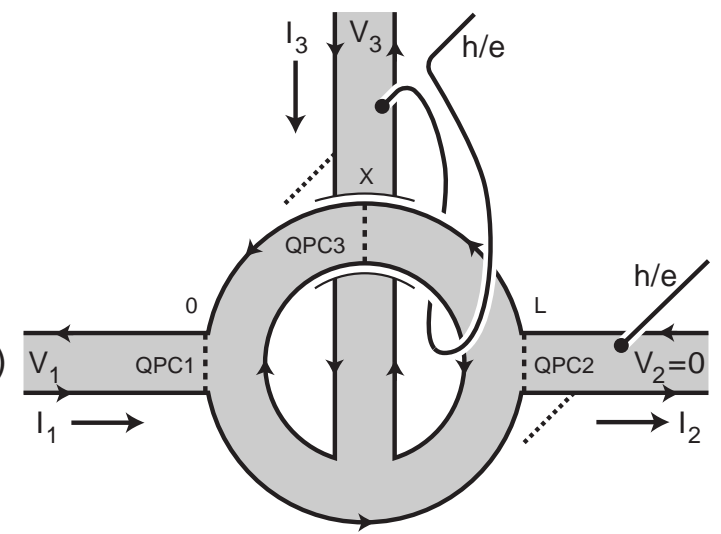

(b)

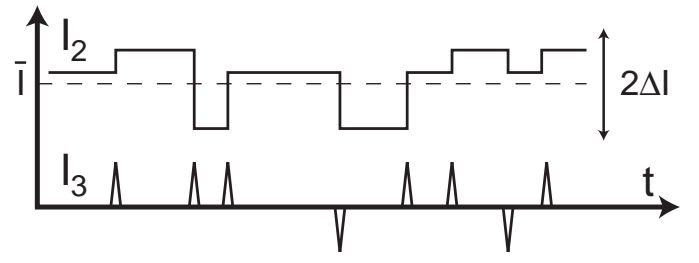

FIG. 1: (a) Schematic of a three terminal AB ring with a third contact in the middle of the ring. The arrows represent the edge state propagation and the dashed lines are QPCs. The third contact has been extended under a bridge, as described in the text. The dots represent LQP's, with flux tubes due to fractional statistics attached. When a LQP passes from lead 2 to lead 3 the effective AB flux in the ring increases by $h / e$, causing the current in lead 2 to switch. (b) The resulting "3 state" telegraph noise in lead 2 predicted for $\nu=1 / 3$ is contrasted with the shot noise in lead 3 .

tween the inner and outer edges of the ring. Lead 3 is on the inner edge. In Fig. 1 we have extended lead 3 under a "bridge". This is useful for modeling the lead as a pair of edges which extend to infinity. We emphasize, however, that this arrangement is topologically equivalent to having the contact physically inside the ring.

The consequences of fractional charge and statistics for equilibrium $\mathrm{AB}$ measurements without the middle lead are well known [8, 9, 10, 13, 14, 15]. A naive argument 
says that the fractional charge $-e^{*}=-e / m$ of the LQP should lead to $\mathrm{AB}$ oscillations with period $h / e^{*}>h / e$. This violates gauge invariance though, since a single flux quantum in the ring can be eliminated with a gauge transformation. The resolution of this apparent paradox is that when the flux through the ring is decreased by $h / e$ the ring is in an excited state with a quasihole of charge $e^{*}$ on the inner edge. In equilibrium, a LQP from outside of the ring will eventually fill the hole. Due to its fractional statistics, the added LQP gives an extra phase $2 \Theta_{m}$ to a LQP circling the ring which exactly cancels the $\mathrm{AB}$ phase, restoring the $h / e$ periodicity. Thus, the absence of multiple AB periods, coupled with the fractional charge of the LQPs constitutes a confirmation of the fractional statistics. But since that absence is guaranteed by gauge invariance, this would hardly make for a satisfying "observation". Chamon et al.[10] have argued $h / e^{*}$ oscillations signifying fractional charge and statistics would be present if the charge on the inner edge could be appropriately adjusted with an electrostatic gate.

Gefen and Thouless 16 have argued that for a ring with a lead in the middle an $\mathrm{AB}$ period $h / e^{*}$ could in principle be observed provided the experiment was done on a sufficiently fast time scale. This is a consequence of the nontrivial topology of the structure, which has a non contractable loop enclosing a contact. This leads to an $m$-fold degeneracy in the FQHE ground state 17] that is intimately related to fractional statistics [18]. Tunneling between the degenerate states occurs when LQPs tunnel through the FQHE fluid between the inner and outer edges of the ring. $h / e^{*} \mathrm{AB}$ oscillations can occur on a time scale faster than the inverse tunneling rate.

Tunneling of LPQ's across QPC3 will lead to temporal fluctuations in the $\mathrm{AB}$ current passing between leads 1 and 2 . This can be seen most transparently by the following topological argument, illustrated in Fig. 1a. Because of their fractional statistics, every LQP is endowed with a "statistical flux tube" seen by other quasiparticles [3]. This represents the statistical phase $2 \Theta_{m}$ acquired when LQPs circle each other as an effective $A B$ phase, and is drawn as a vertical line passing through the LPQ in lead 2 in Fig. 1a. When that LPQ propagates along the top edge to QPC3 and tunnels to the inner edge of the ring the effective flux experienced by LPQs circling the outside of the ring changes by $h / e$. When the LPQ passes under the "bridge" the statistical flux cannot pass through the FQHE state above unless another LPQ tunnels back to the outer edge. The flux gets "hung up" in the ring and persists even after the LPQ has disappeared into lead 3 . With each passing LPQ the effective $\mathrm{AB}$ phase acquired by a LQP encircling the perimeter changes by $2 \Theta_{m}$, causing the $\mathrm{AB}$ current flowing between leads 1 and 2 to switch. This leads to a pattern of fluctuations in the current $I_{2}$ shown in Fig. 1b. $I_{2}$ switches cyclically between $m$ values, which are characteristics of the $m$ degenerate ground states. These fluctuations re- semble random " $m$-state" telegraph noise.

Telegraph noise is a direct consequence of a fractional statistical phase. For sufficiently weak tunneling it could in principle be observed in real time. However, we now show that it also has a distinct signature in the more experimentally accessible low frequency noise. We begin with a simple statistical argument for the noise, which will be justified below with a detailed calculation based on the chiral Luttinger liquid (CLL) model. Suppose that over a time interval $T_{0}$ there are $N$ tunneling events at random times $t_{k}$. Tunneling events in the forward and backward directions, denoted by $s_{k}= \pm 1$, occur with probability $p^{ \pm}$. If the voltage difference between the opposite edges at QPC3 is $V_{3}[19$ and the temperature is $T$, then detailed balance dictates that $p^{+}=p^{-} \exp \left(e^{*} V_{3} / T\right)$. The average tunneling current is then $I_{3}=\left(e^{*} N / T_{0}\right) \tanh e^{*} V_{3} / 2 T$. Here and in the following we set $\hbar=k_{B}=1$.

We assume that the current measured in lead 2 switches when the LQPs tunnel, and has the simple form

$$
I_{2}(t)=\bar{I}+\Delta I \cos \left(\phi_{0}+2 \Theta_{m} \sum_{k} s_{k} \theta\left(t-t_{k}\right)\right) .
$$

Here $\phi_{0}$ is an unspecified phase, and $\theta$ is a step function. Clearly the average value of the current will simply be $\left\langle I_{2}\right\rangle=\bar{I}$. To compute the noise we consider the correlation function, $S(t)=\left\langle I_{2}\left(t_{0}\right) I_{2}\left(t_{0}+t\right)\right\rangle-\bar{I}^{2}$. Averaging over the $N$ times $t_{k}$ and signs $s_{k}$, this may be written as

$$
S(t)=\frac{\Delta I^{2}}{2} \operatorname{Re}\left[\int_{0}^{T_{0}} \frac{d t_{k}}{T_{0}} \sum_{s_{k}= \pm} p^{s_{k}} z_{m}^{s_{k}}\left(\theta\left(t_{0}-t_{k}\right)-\theta\left(t_{0}+t-t_{k}\right)\right)\right]^{N},
$$

where $z_{m}=\exp 2 i \Theta_{m}=\exp 2 \pi i / m$ and $m \neq 1$. In the limit $N, T_{0} \rightarrow \infty$ with $I_{3}$ fixed this becomes

$$
S(t)=\frac{\Delta I^{2}}{2} \operatorname{Re}\left[e^{-\frac{I_{3}|t|}{e^{*}}\left(\operatorname{coth} \frac{e^{*} V_{3}}{2 T}\left(1-\cos 2 \Theta_{m}\right)-i \sin 2 \Theta_{m}\right)}\right] .
$$

For low frequency the noise will be 20 ]

$$
S(\omega \rightarrow 0)=\frac{e^{*} \Delta I^{2}}{2 I_{3}} \frac{\operatorname{coth}\left(e^{*} V_{3} / 2 T\right)}{1+\sin ^{2} \Theta_{m} / \sinh ^{2}\left(e^{*} V_{3} / 2 T\right)} .
$$

For $e^{*} V_{3} \gg T$, all tunneling events will be in the forward direction, and (4) reduces to $S=e^{*} \Delta I^{2} /\left(2 I_{3}\right)$. For $V_{3}=0$ there will be switching due to thermal fluctuations in QPC3, and $S=e^{* 2} \Delta I^{2} /\left(4 G_{3} T \sin ^{2} \Theta_{m}\right)$, where $G_{3}=$ $I_{3} / V_{3}$ is the conductance of QPC3. The frequency dependence of the telegraph noise can easily be determined from (3). For frequency $\omega<\omega_{c}=\left(I_{3} / e^{*}\right) \operatorname{coth}\left(e^{*} V_{3} / 2 T\right)$ it will be frequency independent and given by (4). For $\omega>\omega_{c}, S(\omega)=\Delta I^{2} /\left(2 \omega^{2}\right)$.

In addition to this telegraph noise, there will also be shot noise in the current $I_{2}(t)$. The tunneling of LQPs through QPC3 will generate noise of order $e^{*} I_{3}$, while 
backscattering at $\mathrm{QPC} 1$ and $\mathrm{QPC} 2$ will generate noise of order $e \bar{I}$. Unlike shot noise, however, the telegraph noise varies inversely with the tunneling current $I_{3}$. Thus when $I_{3} \ll \bar{I}, \Delta I$, telegraph noise will be the dominant contribution to the low frequency noise.

Eq. 4, which we established using a heuristic argument, is our central result. We now derive (4) within a CLL model[21]. In addition to providing a concrete theoretical foundation for our assertions, this model calculation gives predictions for the voltage $(V)$ and temperature $(T)$ dependence of $\bar{I}, \Delta I$ and $I_{3}$. The CLL model is a low energy theory, valid in the limit that there is a single edge mode. While real edges may not be in this single channel limit, we suspect that (4) may be of more general validity, since it reflects the topological properties of the bulk LPQ. The detailed $V$ and $T$ dependence however will be sensitive to the single channel assumption.

We focus on the limit in which the LQP backscattering at QPC3 is weak. This is essential for observing telegraph noise, since we require those tunneling events to be uncorrellated. We also consider the LQP backscattering at QPC1 and QPC2 to be weak. While this limit is not necessary for experiment, it allows us to perform a perturbative analysis of the backscattering at QPC1 and QPC2. We compute the currents $I_{2}$ and $I_{3}$ and the corresponding noise as functions of voltages $V_{1}$ and $V_{3}$, with $V_{2}=0$. $I_{2}$ will be $\left(e^{2} / m h\right) V_{1}$ with a small correction due to LPQ backscattering at QPC1 and QPC2. The two backscattering processes will interfere. Without LQP tunneling at QPC3 this leads to AB oscillations with period $h / e^{*}$ in the reflected current. We will show that LPQ tunneling at QPC3 eliminates the equilibrium $\mathrm{AB}$ oscillations and leads to the telegraph noise in Eq. 4.

In the CLL model 21], the low energy edge excitations of the structure in Fig. 1 are described by the Hamiltonian $\mathcal{H}=\mathcal{H}_{1}^{0}+\mathcal{H}_{2}^{0}+\mathcal{H}_{3}^{0}+U_{1}+U_{2}+U_{3} . \mathcal{H}_{i}^{0}$ describes the edge incident from lead $i$,

$$
\mathcal{H}_{i}^{0}=\frac{m v_{F}}{4 \pi} \int d x_{i}\left[\partial_{x} \phi\left(x_{i}\right)\right]^{2} .
$$

$v_{F}$ is the edge state velocity, and the coordinates $x_{i}$ are defined so that at QPC1 $x_{i}=0$, at QPC2 $x_{i}=L$ and at QPC3 $x_{i}=X$. The fields $\phi_{i}\left(x_{i}\right)$ satisfy $\left[\phi_{i}\left(x_{i}\right), \phi_{j}\left(x_{j}^{\prime}\right)\right]=$ $i(\pi / m) \nu_{i} \delta_{i j} \operatorname{sign}\left(x_{i}-x_{j}^{\prime}\right)$, where $\nu_{1}=-\nu_{2}=\nu_{3}=1 \mathrm{spec}-$ ifies the propagation direction.

Tunneling of LQPs at QPC $\alpha$ is described by

$$
U_{\alpha}=v_{\alpha} O_{\alpha}^{+} e^{-i e^{*} \tilde{V}_{\alpha} t}+v_{\alpha}^{*} O_{\alpha}^{-} e^{i e^{*} \tilde{V}_{\alpha} t},
$$

where $v_{\alpha}$ are the complex LQP backscattering matrix elements at $\mathrm{QPC} \alpha$. The relative phase of $v_{1}$ and $v_{2}$ will depend on the magnetic flux in the ring, advancing by $2 \pi / 3$ with every added flux quantum. The exponential factors reflect the voltage difference between the edge states incident on the junction, $\tilde{V}_{1}=\tilde{V}_{2}=V_{1}, \tilde{V}_{3}=V_{3}$.
The LQP backscattering operator for QPC1 is

$$
O_{1}^{ \pm}=\frac{\kappa_{1}^{ \pm}}{(2 \pi \eta)^{1 / m}} e^{ \pm i\left(\phi_{1}(0)-\phi_{2}(0)\right)},
$$

with similar expressions for $O_{2}^{ \pm}$and $O_{3}^{ \pm} \cdot \eta$ is an ultraviolet cutoff, and $\kappa_{\alpha}^{ \pm}$are Klein factors, which are necessary to ensure the correct commutation relations between the operators $O_{\alpha}^{ \pm}[22] . \quad \kappa_{\alpha}^{ \pm}$will play a crucial role in what follows. Their properties may be deduced from the following physical argument. When a LQP backscatters at QPC1, a vortex moves the small distance across the contact. While this will affect the overall phase of the wave function at another part of the sample, it can have no immediate effect on the phase difference across another point contact. Different tunneling operators must therefore commute with one another. Using the commutation relations obeyed by $\phi_{i}\left(x_{i}\right)$ it is then straightforward to establish that the Klein factors must satisfy $\kappa_{\alpha}^{r} \kappa_{\beta}^{s}=\kappa_{\beta}^{s} \kappa_{\alpha}^{r} e^{i r s \xi_{\alpha \beta} \pi / m}$ with $r, s= \pm$. Here $\xi_{\alpha \beta}=-\xi_{\beta \alpha}$ with $\xi_{12}=0$ and $\xi_{13}=-\xi_{23}=1$.

We begin by evaluating the average current $I_{2}=$ $G_{0} V_{1}-I^{b}$. Here $G_{0}=e^{2} / m h$ and $I^{b}$ is the current backscattered by QPC1 and QPC2, which may be written as

$$
I_{b}=\sum_{\alpha=1}^{2}\left\langle T_{C}\left[\hat{I}_{\alpha}\left(t_{0}\right) e^{-i \int_{C} d \tau \sum_{\beta=1}^{3} U_{\beta}(\tau)}\right]\right\rangle_{0} .
$$

$\langle\ldots\rangle_{0}$ denotes a thermal expectation value for $v_{\alpha}=0$. $\hat{I}_{\alpha}(t)=-i e^{*}\left(v_{\alpha} O_{\alpha}^{+} e^{-i e^{*} \tilde{V} t}-\right.$ c.c $)$ are current operators. $C$ is the Keldysh contour, which runs from $-\infty$ to $\infty$ and back. $T_{C}$ specifies time ordering on the Keldysh contour.

Consider first the case $v_{3}=0[10$. Expanding (8) to second order in $v_{1}$ and $v_{2}$ and evaluating the time integrals gives $I_{2}=\bar{I}+\Delta I \cos \phi_{0}$, where

$$
\begin{aligned}
& \bar{I}=G_{0} V_{1}-e^{*}\left(\left|v_{1}\right|^{2}+\left|v_{2}\right|^{2}\right) T^{2 / m-1} F_{1}\left(e^{*} V_{1} / 2 \pi T\right), \\
& \Delta I=2 e^{*}\left|v_{1}\right|\left|v_{2}\right| T^{2 / m-1} F_{2}\left(e^{*} V_{1} / 2 \pi T, 2 \pi T L / v_{F}\right),
\end{aligned}
$$

and $\phi_{0}$ is the phase of $v_{1}^{*} v_{2}$. The first term describes backscattering from a single QPC, with $F_{1}(\tilde{v})=$ $|\Gamma(1 / m+i \tilde{v})|^{2} \sinh \pi \tilde{v} /(\pi \Gamma(2 / m))$. The second term describes the interference between the two QPCs with

$$
F_{2}(\tilde{v}, \tilde{L})=\frac{\Gamma(1 / m-i \tilde{v})\left(e^{2 \pi \tilde{v}}-1\right)}{\pi \Gamma(1 / m)(2 \sinh \tilde{L})^{1 / m}} Q_{1 / m-1}^{i \tilde{v}}(\operatorname{coth} \tilde{L}) .
$$

Here $Q_{q}^{p}(x)$ is the associated Legendre function of the second kind. $F_{2}$ describes how the interference $\Delta I$ is suppressed when $V$ or $T$ is larger than $v_{F} / L$. For fixed $T \lesssim v_{F} / L, \Delta I$ shows oscillations as a function of $V$ with a period $2 \pi v_{F} / e^{*} L . \Delta I$ depends on magnetic field, and predicts $\mathrm{AB}$ oscillations with period $h / e^{*}$. To remedy that error we must include $v_{3}$. Though $v_{3}$ is small, it will be necessary to expand (8) to all orders in $v_{3}$. 
The perturbative expansion of (8) in powers of $v_{3}$ is similar to the partition function for a collection of charged "particles", which correspond to tunneling events at different times 23. Forward and backward tunneling events have opposite charge. At finite temperature, positive and negative charges will be confined in pairs, separated by a time of order $1 / T$. Physically, these pairs correspond to real tunneling events when they occur on opposite Keldysh paths and virtual tunneling events when they are on the same path. When $v_{3} T^{1 / m-1} \ll 1$, those pairs will be dilute and never overlap. In that limit it is straightforward to expand $I_{b}$ to all orders in $v_{3}$.

The $v_{3}^{2 N}$ term in the expansion involves a set of $N$ pairs of charges. The positive (negative) charges are at $t_{k}^{ \pm}=t_{k} \pm \delta t_{k} / 2$, for $k=1 \ldots N$. There are two opposite charges coming from $v_{1}$ and $v_{2}$ at $t_{0}^{ \pm}=t_{0} \pm \delta t_{0} / 2$. To keep track of the forward and backward paths in this expansion, we introduce an index $\sigma_{k}^{ \pm}= \pm 1$ such that $\tau_{k}^{ \pm} \equiv\left(t_{k}^{ \pm} ; \sigma_{k}^{ \pm}\right)[24]$. The $2 \mathrm{Nth}$ term in the expansion will involve an expectation value of the form

$$
\Pi=\left\langle T_{C}\left[O_{\alpha}^{+}\left(\tau_{0}^{+}\right) O_{\beta}^{-}\left(\tau_{0}^{-}\right) \prod_{k=1}^{N} O_{3}^{+}\left(\tau_{k}^{+}\right) O_{3}^{-}\left(\tau_{k}^{-}\right)\right]\right\rangle .
$$

This can be evaluated as the product of two terms. The first comes from the expectation value of the Bose fields $\phi_{i}$. When the pairs are well separated, so that $T\left|t_{k}-t_{l}\right| \gg$ 1 we find that this can be written as a product over all pairs, $\Pi_{1}=G_{\alpha \beta}^{\sigma_{0}^{+} \sigma_{0}^{-}}\left(\delta t_{0}\right) \prod_{k=1}^{N} G_{33}^{\sigma_{k}^{+} \sigma_{k}^{-}}\left(\delta t_{k}\right)$, where the $G$ 's are correlation functions within a single pair. The second term comes from the time ordered product of the Klein factors, and is given by $\Pi_{2}=\prod_{k=1}^{N} z_{m}^{\theta\left(t_{0}-t_{k}\right) \zeta_{\alpha \beta}\left(\sigma_{k}^{+}-\sigma_{k}^{-}\right) / 2}$, where $\zeta_{\alpha \beta}=\left(\xi_{\alpha 3}-\xi_{\beta 3}\right) / 2$. Since $\Pi$ factorizes into a product over $k$ of $N$ identical terms, the expansion in powers of $v_{3}$ can be resummed. Suppose we turn on $v_{3}$ at time $t=0$. We then find $I_{b}=\sum_{\alpha \beta} I_{\alpha \beta}$ with

$$
I_{\alpha \beta}=I_{\alpha \beta}^{0} e^{-\frac{I_{3} t_{0}}{e^{*}}\left(\operatorname{coth} \frac{e^{*} V_{3}}{2 T}\left(1-\cos \frac{2 \pi \zeta_{\alpha \beta}}{m}\right)+i \sin \frac{2 \pi \zeta_{\alpha \beta}}{m}\right)} .
$$

$I_{\alpha \beta}^{0}$ is the $v_{\alpha} v_{\beta}^{*}$ term in the expansion of (8) for $v_{3}=$ 0 , and $I_{3}=e^{*} v_{3}^{2} T^{2 / m-1} F_{1}\left(e^{*} V_{3} / 2 \pi T\right)$. This shows that the interference terms average away after a time $\tanh \left(e^{*} V_{3} / 2 T\right) / I_{3}$ due to LQP tunneling, leaving only the diagonal terms, $I_{2}=\bar{I}$.

The noise in lead 2 is calculated by computing $S(t)=$ $\left\langle\left\{I_{2}\left(t_{0}+t\right), I_{2}\left(t_{0}\right)\right\}\right\rangle / 2-\bar{I}^{2}$. There will be several contributions. At order $v_{1,2}^{2}$ there will be the "single barrier" shot noise [5] produced by QPCs 1 and 2 given by $e^{*} I_{b}$ for $e^{*} V_{3} \gg T$. Shot noise produced by QPC3 will also be present at order $v_{1}^{2} v_{3}^{2}$. The telegraph noise comes in at order $v_{1}^{2} v_{2}^{2}$. The $v_{3}$ expansion is similar to that above, and leads precisely to (4), which is of order $v_{1}^{2} v_{2}^{2} / v_{3}^{2}$.

Optimal experimental conditions for observing telegraph noise include, (1) $I_{3} \ll G_{0} V_{3}$, so that LQPs are dilute. (2) $I_{3} \ll \Delta I$, so that telegraph noise dominates shot noise. It is desirable to set QPC1 and QPC2 so that they backscatter moderately to maximize the interference signal. It is also necessary that (3) $V_{1}, T \lesssim v_{F} / L$, or else the interference will be washed out. Finally, the frequency of the measured noise should be low enough so that $(4) \omega \lesssim\left(I_{3} / e^{*}\right) \operatorname{coth} e^{*} V_{3} / 2 T$.

We now comment briefly on the generalization of the above results to hierarchical states. For $\nu=1 / m$ the quasiparticle charge, statistics and Hall conductivity all have the same numerical value, and can be argued to reflect the same quantity. By contrast, for hierarchical states these quantities differ. For example $\nu=2 / 7$ has quasiparticles with charge $-e / 7$ and statistics $\Theta=3 \pi / 7$. Both the heuristic arguments and the CLL theory can be generalized to account for hierarchical states, and Eq. (4) is recovered with the appropriate statistics angle $\Theta$.

It is a pleasure to thank Moty Heiblum for helpful discussions.

[1] R.B. Laughlin, Phys. Rev. Lett. 50, 1395 (1982).

[2] B.I. Halperin, Phys. Rev. Lett. 52, 1583 (1984).

[3] D. Arovas, J.R. Schrieffer and F. Wilczek, Phys. Rev. Lett. 53, 722 (1984).

[4] F. Wilczek, Phys. Rev. Lett. 49, 957 (1982).

[5] C.L. Kane and M.P.A. Fisher, Phys. Rev. Lett. 72, 724 (1994).

[6] R. de-Picciotto, et al., Nature 389, 162 (1997).

[7] L. Saminadayar, et al., Phys. Rev. Lett. 79, 2526 (1997).

[8] S.A. Kivelson, Phys. Rev. Lett 65, 3369 (1990).

[9] J.K. Jain, S.A. Kivelson and D.J. Thouless, Phys. Rev. Lett. 71, 3003 (1993).

[10] C. Chamon, et al., Phys. Rev. B 55, 2331 (1997).

[11] I. Safi, P. Devillard and T. Martin, Phys. Rev. Lett. 86, 4628 (2001).

[12] Y. Ji, et al., Nature 422, 415 (2003).

[13] J.A. Simmons, et al., Phys. Rev. Lett. 63, 1731 (1989).

[14] P.A. Lee, Phys. Rev. Lett. 65, 2206 (1990).

[15] V.J. Goldman and B. Su, Science 267, 1010 (1995).

[16] D.J. Thouless and Y. Gefen, Phys. Rev. Lett. 66, 806 (1991); Y. Gefen and D.J. Thouless Phys. Rev. B 47, 10423 (1993).

[17] D.J. Thouless, Phys. Rev. B 40, 12034 (1989).

[18] X.G. Wen and Q. Niu, Phys. Rev. B 41, 9377 (1990).

[19] When backscattering at QPC2 is not weak, this will be the difference between $V_{3}$ and an appropriate average of $V_{1}$ and $V_{2}$.

[20] This differs by a factor of 2 from the conventional definition, which for classical shot noise gives $S=2 e I$. With our definition $S=e I$.

[21] X.G. Wen, Phys. Rev. Lett. 64, 2206 (1990).

[22] R. Guyon, et al., Phys. Rev. B 65153304 (2002).

[23] C. Chamon, D. Freed and X.G. Wen, Phys. Rev. B 51, 2363 (1995).

[24] C.L. Kane and M.P.A. Fisher, Phys. Rev. B 67, 045307 (2003). 